\title{
Blue whale (Balaenoptera musculus musculus) genome: population structure and history in the North Atlantic
}

\author{
Sushma Jossey ${ }^{1}$, Oliver Haddrath ${ }^{1}$, Livia Loureiro ${ }^{2}$, Burton Lim ${ }^{1}$, Jacqueline Miller ${ }^{3}$, Si \\ Lok $^{2}$, Stephen Scherer ${ }^{2}$, Anders Goksoyr ${ }^{4}$, Roger Lille-Langøy ${ }^{4}$, K Kovacs ${ }^{5}$, Christian \\ Lydersen $^{5}$, Heli Routti ${ }^{5}$, and Mark Engstrom ${ }^{1}$ \\ ${ }^{1}$ Royal Ontario Museum \\ ${ }^{2}$ The Hospital for Sick Children \\ ${ }^{3}$ Royal Ontario Museum Department of Natural History \\ ${ }^{4}$ University of Bergen \\ ${ }^{5}$ Norwegian Polar Institute
}

April 14, 2021

\begin{abstract}
Knowledge of genetic diversity and structure is essential for developing conservation strategies for endangered species. The advances in museum genomics can assist in better understanding the effects of over-hunting on the genome by comparing historical to present-day samples. Blue whales were hunted to the point of near extinction in the mid-twentieth century. Herein, we use whole genome sequencing to elucidate the poorly understood population structure of North Atlantic (NA) blue whales (Balaenoptera musculus musculus). We generated a de novo genome assembly of $2.49 \mathrm{Mbp}$ for a NA blue whale (N50 of $1.46 \mathrm{Mb}$ ) to analyze 19 whole genomic sequences and 28 complete mitochondrial genomes. We included present-day and historical samples (earliest from 1900) from the Atlantic and Antarctica to understand the impact of whaling on the genetic diversity. We found low population structuring, but high genetic diversity, suggesting a single, panmictic population in the NA. We identified gene flow from fin whale to blue whales, accounting for $\sim 3.5 \%$ of the genome. Introgression between blue and fin whales was observed in all the present-day samples but were lacking in some whales sampled early in the 20th century, which suggests increasing disruption in mate choice concomitant with decline in blue whale population. We also assembled and analyzed the transcriptome and revealed positive selection of oncogenes, which may be involved in reduced cancer rates in this largest of mammals ever known. Our sequencing and population structuring studies provide a genomic framework to guide ongoing conservation strategies for this iconic species.
\end{abstract}

\section{INTRODUCTION}

Many wildlife populations are currently under threat from climate change, pollution, loss of habitat and in some cases exploitation. Genomic approaches in non-model organisms are becoming an important tool to study population structure and history that can help protect endangered species (Hohenlohe et al., 2020). The analysis of whole genomes can help provide detailed information on demographic changes, genetic diversity, detection of introgression in organisms (Miller et al., 2012) and the population structure in species with wide ranges (Foote et al., 2019; Sinding et al., 2018). These genomic tools are also ideal to develop an understanding of global population histories in whales with limited barriers to movement across the oceans (Foote et al., 2019). Temporal sampling of genomic data from historical specimens held in museum collections can provide valuable baselines to accurately quantify genetic threats and can help decipher genomic changes in endangered species (Díez-del-Molino et al., 2018; Ewart et al., 2019; Mikheyev, Tin, Arora, \& Seeley., 2015). Advances in genomic approaches can moreover help identify genes that affect the fitness of a species 
in relation to anthropogenic threats (Meyer et al., 2018).

The blue whale (Balaenoptera musculus musculus ) is the largest animal that has ever lived, with individuals reaching $30 \mathrm{~m}$ in length and weighing up to 150 tonnes (Sears \& Perrin, 2018). Blue whales are found in oceans across the globe, but were historically most abundant in the Southern Ocean (Sears \& Perrin, 2018). Commercial hunting of these whales started in the North Atlantic (NA) in 1860 and spread to all other oceans (Thomas et al., 2016). The large size of blue whales made them a prime target for the commercial whaling industry during the 20th century and they were hunted to the brink of extinction with an estimated 379,185 whales taken globally (Rocha et al., 2014). Whether blue whales in the NA represent a single population or two distinct populations, separated between the west and east is unresolved (Lesage et al., 2016; McDonald et al., 2006). The Northwest Atlantic (NWA) has a lower number of blue whales, with some 250 adults (COSEWIC, 2012 ), whereas in the Northeast Atlantic (NEA) there are ${ }^{\sim} 3000$ individuals (Pike et al., 2019 ). The movement patterns of blue whales in the NA and the location(s) of their wintering sites and breeding areas are still elusive (Lesage et al., 2017). The blue whales' distribution in the NWA, based on sightings and photo-identification studies, ranges from the mid Atlantic westward to the Davis Strait and west coast of Greenland in the north and southward along the eastern coasts of Canada and USA (COSEWIC, 2012; Lesage et al., 2017). In the NEA, blue whale sightings occur from Svalbard, Norway (Storrie et al. 2018) in the north to the Azores in the south, with rare sightings off Mauritania (Pike et al. 2019). Vocalization records indicate the presence of blue whales near the Mid-Atlantic Ridge in winter (Nieukirket al., 2004), but it is not known whether these animals are from the NEA or NWA. The small number of NA blue whales that have been followed using satellite tracking show no evidence of east-west movements across the North Atlantic (Lesage et al., 2017; Silva et al., 2013). Comparison of blue whale photo-identifications taken near Iceland and the Azores (NEA) to NWA blue whales also suggests two distinct populations in the Northwest and Northeast (Lesage et al., 2017; Ramp \& Sears, 2013). However, blue whale songs recorded from the NEA and NWA are similar to each other and distinct from blue whales in other oceans, suggesting that the NA may represent a single population (McDonald et al., 2006).

Blue whale bones exposed to the elements have been used to extract mitochondrial DNA (Tebbutt et al., 2000) and the potential for historical blue whale bone samples t can help decipher population structure (Rosenbaum et al., 1997), as well as to study changes in the genome, from changes in levels of heterozygosity to interspecific hybridization among other things. Purported blue whale/fin whale hybrids were reported by early whalers from the coast of Norway, Gulf of Alaska, Icelandic waters and the coast of Spain (Pampoulie et al., 2020). In the last few decades, the identification of hybrids has been conclusively demonstrated using molecular evidence (Pampoulie et al., 2020). But it is not clear if this hybridization has led to significant gene flow between the two species (Westbury et al., 2019). Significant levels of gene flow from fin whales (population size $>80,000$, Pampoulie et al., 2020) to blue whales could represent a threat to the maintenance of a genetically distinct NA population(s) of blue whales.

In the NA, blue whales are classified as being Endangered in Europe (IUCN Red List, Pampoulie et al., 2020) and in North America they are listed under the Canadian Species at Risk Act and the US Endangered Species Act (Lesage et al., 2017). Blue whales are threatened by ship strikes, fishing gear entanglement, marine pollutants and the impacts of climate change (COSEWIC, 2012). Knowledge of the genetic structure and distribution of blue whales in the NA is essential for the protection of this vulnerable species. Challenges to the study of movement of large marine mammals includes attaching instruments to these fast swimming animals and retaining the tracking devices on the animals long enough to document seasonal patterns (Lesage et al., 2017). This has left a gap in our understanding of NA blue whale population structuring. Therefore, the principal goals of this study were to construct a highly accuratede novo assembly of a NWA blue whale genome and to use it to study our unique collection of present-day and historical specimens to gain insights into population structure, introgression, historic population size and dynamics of the world's largest animal.

\section{MATERIALS AND METHODS}

\subsection{Genome assembly and annotation}


Muscle tissue for the genome assembly was collected by the Royal Ontario Museum (ROM), Toronto, ON, with the approval of the Minister of Fisheries and Oceans, Canada (SARA permit ref: NLSAR-003-14) from a female blue whale that died close to Newfoundland in 2014 (NW-M6, Fig. 1, Table 1). The Illumina and Pacbio reads for the genome assembly were generated at The Centre for Applied Genomics (TCAG), The Hospital for Sick Children, Toronto, Canada. The genome was assembled using the hybrid assembler MASURCA v 3.2.8 (Koren et al., 2012; see Supplemental Information for more details).

RNA for the transcriptome assembly was collected from a skin biopsy of a blue whale sampled in the Svalbard Archipelago $\left(79^{\circ} \mathrm{N}\right)$, Norway (Fig. 1). The paired-end RNAseq data were generated using HiSeq 2500 at TCAG. The transcripts were assembled with TRINITY (Grabherr et al., 2011) and TOPHAT (Trapnell et al., 2009) as elaborated in Supplemental Information. The masked genome was annotated using the MAKER2 (Holt \& Yandell, 2011) pipeline with the blue whale transcriptome, NCBI proteins for cow and all cetaceans as explained in Supplemental Information. Functional annotations of the predicted genes were done by BLASTp (Altschul et al., 1997) hits to UNIPROT (UniProt Consortium 2015) using an E-value of $<1 \mathrm{e}-6$.

\subsection{Positive Selection}

To identify genes in the blue whale, the largest mammal, that are positively selected and associated with longevity or reduced cancer rates, we compared genomes of the blue whale with one of the smallest baleen whales, the minke whale (Balaenoptera acutorostrata, Yim et al., 2014). The NA blue whale and the minke whale transcripts were filtered and translated. The single gene orthologs (SGOs) were identified between blue and minke whales with reciprocal best hits using VESPA (Webb et al., 2017), BLASTp and MUSCLE (Edgar, 2004). Positive selection was analyzed using pairwise comparison with KAKS_CALCULATOR v2.0 (Wang, Zhang, Zhang, Zhu, \& Yu, 2010) between NA blue whale transcript and minke whale (downloaded from NCBI: GCF_000493695.1_BalAcu1.0). Tajima's D (Sabeti et al., 2006) analysis was used to screen for regions under positive selection for the 12 present-day NA blue whales (Supplemental Information). The paired-end Illumina reads from the 12 whales were aligned to masked autosomal genome of the NA blue whale and the SNPs detected were analyzed as described in the Supplemental Information.

\subsection{Whole genome sequencing}

Present-day (from whales after 2010) and historic (from blue whales that died during 1900-1975) samples were included in our analyses to explore the effects of whaling. The reference-based alignments of the 12 blue whales (11 sequenced here and the genomic blue whale sequences from Árnason et al., (2018) represented here as NE-Ar) from present-day were performed to the masked autosomal scaffolds of the assembled NA blue whale genome and used in the population structure, heterozygosity estimation and introgression analyses. DNA from historical blue whale bone samples were collected from: the ROM; Canadian Museum of Nature, Ottawa, Ontario; Smithsonian National Museum of Natural History, Washington D.C.; Slottsfjells Museum, Tønsberg, Norway; and The Whaling Museum, Sandefjord, Norway (Fig. 1, Table 1). DNA extraction was carried out in an ancient DNA facility at ROM following the methodology detailed in Supplemental Information. The paired-end reads were sequenced on an Illumina HiSeq X sequencer at TCAG and the adapter sequences were trimmed and paired-end reads were merged using Seqprep v 1.1 (https://github.com/jstjohn/SeqPrep) with default settings and quality score cut-off for mismatches in overlap $>20$ (-q 20). The first and last two bases of the merged reads were trimmed to remove DNA damage in historical bone samples. The paired-end reads were aligned using BWA 0.7.17 ( $\mathrm{Li} \&$ Durbin, 2009) and variant discovery was conducted as described in the Supplementary Information. 


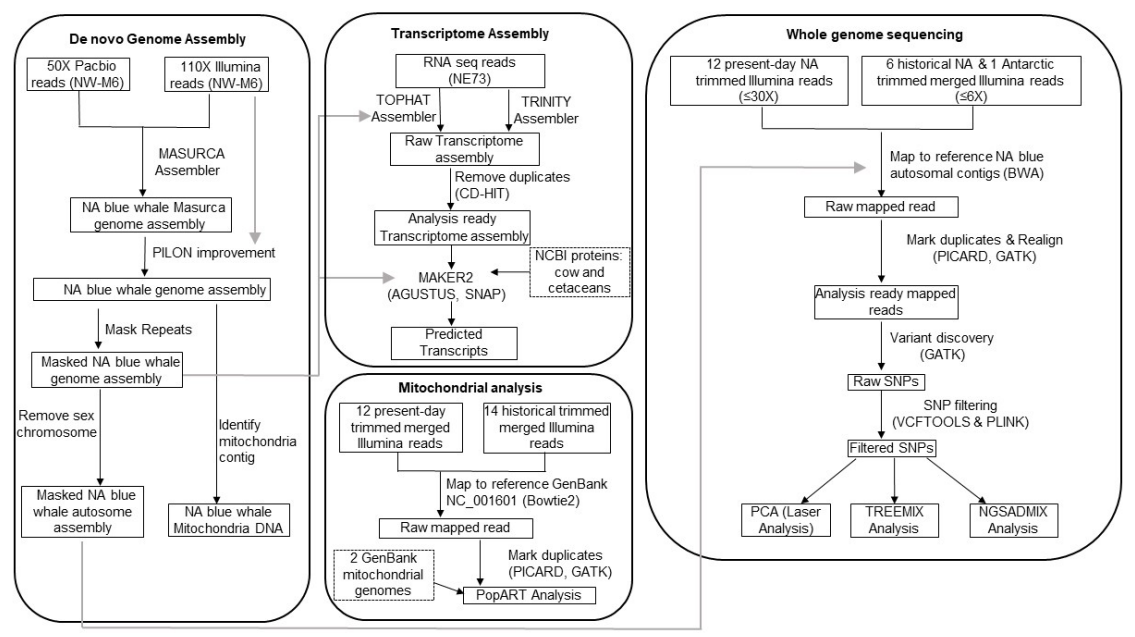

Fig. 1. A flow chart representing the genome assembly, transcriptome assembly, whole genome and complete mitochondrial genome analysis of North Atlantic blue whales.

Table 1. Present day and historical museum blue whale samples with the average coverage (short reads) compared to the repeat masked autosomal North Atlantic nuclear (N) or mitochondrial (M) genome.

\begin{tabular}{|c|c|c|c|c|c|}
\hline Samples & Collection and Collection Number & Collection Locality & Collection Date & Gender & Coverage Nuc \\
\hline NW-M6 & ROM $^{\mathrm{P}} ; \mathrm{ROMM} 125066$ & Newfoundland, Canada & 2014 & Female & 99X $(\mathrm{N})$ \\
\hline NW-B7 & $\mathrm{ROM}^{\mathrm{P}} ; \mathrm{ROMM} 125065$ & Newfoundland, Canada & 2014 & Female & $33 \mathrm{X}(\mathrm{N}) ; 71 \mathrm{k}$ \\
\hline NW7 & $\mathrm{ROM}^{\mathrm{P}} ; \mathrm{MARS} 2017$ & Newfoundland, Canada & 2017 & Female & $19 \mathrm{X}(\mathrm{N}) ; 45 \mathrm{k}$ \\
\hline NW9 & $\mathrm{ROM}^{\mathrm{P}} ; \mathrm{MARS} 2019$ & Nova Scotia, Canada & 2019 & Male & $28 \mathrm{X}(\mathrm{N}) ; 8.5 \mathrm{l}$ \\
\hline NWa-R4 & $\mathrm{ROM}^{\mathrm{H}} ; 108281$ & Magdalen Islands, Canada & 1931 & Unknown & $3 \mathrm{X}(\mathrm{N}) ; 390$ \\
\hline NWa1 & $\mathrm{NMNH}^{\mathrm{H}} ;$ USNM124326 & Newfoundland, Canada & 1903 & Male & $32 \mathrm{X}(\mathrm{M})$ \\
\hline NWa2 & NMNH ${ }^{\mathrm{H}}$; USNMA49784 & Newfoundland, Canada & 1903 & Female & $82 \mathrm{X}(\mathrm{M})$ \\
\hline NWa3 & NMNH ${ }^{\mathrm{H}}$; USNMA49785 & Newfoundland, Canada & 1903 & Unknown & $5 \mathrm{X}(\mathrm{N}) ; 956$ \\
\hline NWa4 & NMNH ${ }^{\mathrm{H}}$; USNMA49786 & Newfoundland, Canada & 1903 & Unknown & $3 \mathrm{X}(\mathrm{N}) ; 338$ \\
\hline NWa5 & NMNH ${ }^{\mathrm{H}}$; USNMA49787 & Newfoundland, Canada & 1903 & Male & $2 \mathrm{X}(\mathrm{N}) 1.4 \mathrm{kX}$ \\
\hline NWa6 & NMNH ${ }^{\mathrm{H}}$; USNMA49788 & Newfoundland, Canada & 1903 & Male & $0.5 \mathrm{X}(\mathrm{N}) 1.4 \mathrm{k}$ \\
\hline NWa-CM1 & $\mathrm{CMN}^{\mathrm{H}} ; \mathrm{CMNMA55059}$ & Newfoundland, Canada & 1974 & Unknown & $3 \mathrm{X}(\mathrm{N}) 60 \mathrm{X}(\mathrm{I}$ \\
\hline NWa-CM2 & $\mathrm{CMN}^{\mathrm{H}} ; \mathrm{CMNMA}^{2} 5060$ & Newfoundland, Canada & 1975 & Male & $56 \mathrm{X}(\mathrm{M})$ \\
\hline NWa-CM3 & $\mathrm{CMN}^{\mathrm{H}} ; \mathrm{CMNMA56549}$ & Newfoundland, Canada & 1966 & Female & $264 X(\mathrm{M})$ \\
\hline NE1 & $\mathrm{NPI}^{\mathrm{P}} ; \mathrm{BM} 1401$ & Svalbard, Norway & 2014 & Female & $18 \mathrm{X}(\mathrm{N}) 2.2$ \\
\hline NE2 & $\mathrm{NPI}^{\mathrm{P}} ; \mathrm{BM} 1602$ & Svalbard, Norway & 2016 & Male & $36 \mathrm{X}(\mathrm{N}) 15$. \\
\hline NE3 & $\mathrm{NPI}^{\mathrm{P}} ; \mathrm{BM} 1603$ & Svalbard, Norway & 2016 & Male & $19 \mathrm{X}(\mathrm{N}) 2.2$ \\
\hline NE4 & $\mathrm{NPI}^{\mathrm{P}} ; \mathrm{BM} 1604$ & Svalbard, Norway & 2016 & Female & 21X (N) 13.9 \\
\hline NE71 & $\mathrm{NPI}^{\mathrm{P}} ; \mathrm{BM} 1701$ & Svalbard, Norway & 2017 & Male & $4 \mathrm{X}(\mathrm{N}) 1.4 \mathrm{kX}$ \\
\hline NE72 & $\mathrm{NPI}^{\mathrm{P}} ; \mathrm{BM} 1702$ & Svalbard, Norway & 2017 & Female & $18 \mathrm{X}(\mathrm{N}) 2.8$ \\
\hline NE73 & $\mathrm{NPI}^{\mathrm{P}} ; \mathrm{BM} 1703$ & Svalbard, Norway & 2017 & Male & $19 \mathrm{X}(\mathrm{N}) 2 \mathrm{kX}$ \\
\hline NE-Ar & SRR5665644 ${ }^{\mathrm{P}}$ & Iceland & & Unknown & $22 \mathrm{X}(\mathrm{N}) 22 \mathrm{k}$ \\
\hline NEa-I2 & $\mathrm{SlM}^{\mathrm{H}} ; \mathrm{TB} 2$ & Iceland & 1900 & Male & $7.7 \mathrm{X}(\mathrm{M})$ \\
\hline SAa1 & $\mathrm{WM}^{\mathrm{H}} ; \mathrm{HS} 0171$ (SF1) & South Atlantic & $1934 / 35$ & Unknown & $475 \mathrm{X}(\mathrm{M})$ \\
\hline Atla1 & $\mathrm{WM}^{\mathrm{H}} ; \mathrm{HS} .01102(\mathrm{SF} 2)$ & Atlantic Ocean & pre-1917 & Male & $15 \mathrm{X}(\mathrm{M})$ \\
\hline Antarctic & $\mathrm{NMNH}^{\mathrm{H}} ; \mathrm{USNM} 268731$ & Antarctica & 1938 & Female & $6 \mathrm{X}(\mathrm{N}) 219 \mathrm{X}$ \\
\hline
\end{tabular}


NW Northwest Atlantic

NE Northeast Atlantic

SA South Atlantic

P Present day sample from frozen tissues

${ }^{\mathrm{H}}$ Historical sample from bone samples

ROM Royal Ontario Museum, Toronto, ON, CA.

NMNH Smithsonian National Museum of Natural History, Washington DC.

CMNMA Canadian Museum of Nature, Ottawa, ON.

NPI Norwegian Polar Institute, Troms $\varnothing$, Norway

SlM Slottsfjells Museum, Tønsberg, Norway

WM The Whaling Museum, Sandefjord, Norway

\subsection{Mitochondrial genome assembly}

For the comparisons of the mitochondrial genomes of present-day and historical blue whales, the trimmed and merged Illumina paired-end reads for all samples were aligned to a reference mitogenome (NC_00160136) using BOWTIE2 2.3.3.1 (Langmead \& Salzberg, 2012). This was done for 25 of the blue whales sequenced herein (Table 1), including individuals for which DNA was too degraded for whole genome sequencing.

\subsection{Population structure analysis}

To study if blue whales in the NWA represent a separate population from the NEA, genomic population structure analyses were conducted on present-day tissue samples of four NWA (NW-M6, NW-B7, NW7, NW9) and eight NEA (NE1, NE2, NE3, NE4, NE71, NE72, NE73, NE-Ar) blue whales. One sample (NWM6) with high coverage of short read (99X) was adjusted to 30X for this analysis to help SNP filtering and avoid any bias due to large differences in depth of coverage. To deduce the population structure of the NA blue whales prior to whaling and contrast it to present-day population structure, six NWA (NWa-R4, NWa3, NWa4, NWa5, NWa6, NWa-CM1) blue whales dating from the early days of blue whale whaling through to post-whaling and one Antarctic blue whale (Table 1) were included in the analyses. Population structure was investigated using principal component analysis (PCA) and other population structure analyses such as TREEMIX (Pickrell \& Pritchard, 2012). and NGSADMIX (Skotte et al., 2013).

\subsubsection{Principal component analysis}

PCA was performed using LASER v 2.04 (Wang et al., 2015) which uses projection Procrustes analysis for the samples with low depth of coverage were placed in the context of a reference PCA space constructed using genotypes of a set of reference individuals with higher coverage depth. The first PCA analysis with blue, fin and sei whale samples which included $18 \mathrm{NA}$ blue whales (four present-day and six historical samples from NWA, and eight present-day samples from NEA); one historical sample from an Antarctic blue whale (Table 1); seven present-day fin whales from NA and two sei whales (SRR5665645 and SRR5665646). Historical samples, NWa-R4, NWa3, NWa4, NWa5, NWa6, NWa-CM1 were included in this analysis. The second PCA analysis visualized the genetic relationship among blue whales, which included 12 present-day blue whales sampled from both sides of the NA, three historical samples from the NWA (NWa-R4, NWa3 \& NWa4) and the historical sample from Antarctica. The trimmed sequences of the blue, fin and sei whales were reference aligned to the assembled NA blue whale genome autosomes, variant detected and VCF format files were generated. The biallelic SNPs for the PCA were filtered for sites present in at least $50 \%$ of the samples, $>10$ bases apart, with a quality score of $>30$, mapping quality $>30$, coverage depth of between $3 \mathrm{X}$ and $130 \mathrm{X}$ and MAF of $>0.1$. The sites were filtered for linkage disequilibrium by eliminating sites with a correlation coefficient $\left(\mathrm{r}^{2}\right)>0.8$ within a $1 \mathrm{~kb}$ window and 4,136,458 and 2,620,383 sites were used for the first and second 
PCA analysis, respectively. The ancestry reference PCA space for the first PCA analysis was constructed using 11 present-day NA blue whales with $>20 \mathrm{X}$ coverage (Table 1), two present-day fin whales with $>20 \mathrm{X}$ coverage and two sei whales with $\sim 10 \mathrm{X}$ coverage. Four NWA and six NEA present-day blue whales with high coverage and a sei whale (SRR5665645) were included to compute the reference PCA space for the second PCA analysis.

\subsubsection{Treemix}

Genetic relationships were visualized for the 12 present-day blue whale samples from the NA, three historical samples from the NA and one historical sample from Antarctica using TREEMIX v1.13 setting a NA fin whale as the root. The blue whale samples included here showed low levels of introgression from fin whales. Each individual whale was plotted with a maximum likelihood graph (with option -nos) using genome-wide allele frequencies and a Gaussian distribution to account for genetic drift, allowing for two migration events. Treemix was run independently 10 times to verify that the edges were consistent across runs. Migration edges were fitted iteratively by analyzing the residual plots and confirming gene flow events with D-statistics.

\subsubsection{NGSadmix}

The individual admixture proportions for the blue whale samples from the Treemix analysis were estimated by NGSADMIX, a maximum-likelihood method based on genotype likelihoods in ANGSD v0.9 (Korneliussen et al., 2014). NGSADMIX was run with clusters (k) of 2-5, and each k value was run 10 times to look for convergence. The variant sites were filtered to include only those covered in at least 11 individuals with a SNP p-value of $<1 \mathrm{e}-6$ and a MAF $>0.01$.

The genetic divergence between blue whales in the NA was also investigated using Weir and Cockerham weighted Fst (Weir \& Cockerham, 1984). The Fst was estimated with 21,81,260 SNPs filtered as in the PCA analysis for four NWA and seven NEA present-day samples with at least $>20 \mathrm{X}$ coverage.

\subsubsection{Network Analysis}

Haplotype median-joining networks were created using the program POPART (Leigh \& Bryant, 2015) with the default settings. This was carried out for 28 whole mitochondrial genomes including 14 from the NWA (four present-day and ten historical samples), eleven from the NEA (one historical, seven present-day from this study, and three from GenBank MF409242, X72204 and assembled from SRR5665644), a South Atlantic historical sample, an Antarctic historical sample and one of uncertain North Atlantic origins.

The median-joining network for the control region was examined for the 28 samples described above along with an additional 126 mitochondrial partial control region sequences available in GenBank (Supplementary Information). Additional haplogroups available from GenBank were not included due to their shorter sequence length as the algorithm collapses sites that are missing or ambiguous. The consensus length of the control region sequence examined was 413 base pairs.

\subsection{Heterozygosity}

To estimate heterozygosity, ten present-day NA blue whale samples with sequence coverage of $>20 \mathrm{X}$ were aligned to masked autosomal contigs of NA blue whales as described above and the resulting alignments were analyzed with default settings of MLRHO (Haubold et al., 2010). MLRHO gives a maximum likelihood estimate of the population mutation rate $(4 \mathrm{Ne} \mu)$ from individual whole genome sequencing data, which approximates expected heterozygosity under the infinite sites model. The heterozygosity of the Antarctic historical sample $(\sim 6 \mathrm{X})$ was determined using ANGSD based on site frequency spectrum (SFS) using infinite sites model. The trimmed paired-end reads from the Antarctic sample were also aligned to the NA blue whale masked autosomal contigs and ANGSD analysis was performed with filtering for quality score of $>20$, mapping quality $>20$ and options: -noTrans (to remove deaminated cytosine residues in historical samples) and -fold 1.

\subsection{Pairwise sequentially Markovian coalescent (PSMC) model}


The PSMC model was used to estimate changes in effective population size (Ne) through time (Li \& Durbin, 2012). To infer population size history, whole genome sequences of an individual from the NWA (NW-B7) and whales from the NEA (NE2 and NE-Ar) were aligned to the masked NA blue whale assembly. The trimmed reads were aligned with BWA 0.7.17 and bam format files were generated following GATK best practices. A fin whale $(\sim 20 \mathrm{X})$ was also included to infer its population history. The diploid consensus file for each individual was generated using SAMTOOLS MPILEUP version 1.5 following Li and Durbin (2012), and minimum and maximum depth of coverage thresholds were set to between 10X and 100X for all samples. The software PSMC v 0.6.5 was used with plots scaled by the mutation rates and generation times for the two different species of whales from Árnason et al., (2018).

\subsection{Introgression}

Gene flow between blue and fin whale genomes was investigated for present-day and historical samples and gene flow between blue whales and humpback/sei whale genomes was also explored. Sei whales are the most closely related species to blue whales, and humpback whales are the closest related species to fin whales based on the species tree by Árnason et al. (2018). The gene flow between these large baleen whales was examined using D-statistics (Green et al., 2010) in the present-day and historical whale samples to assess if there have been any changes in frequency of hybridization and introgression through time.

The D-statistics or ABBA/BABA test was used to study introgression between blue and fin whales. A fourtaxon phylogeny of (((Antarctic Blue, NA Blue), Fin), Minke) was analyzed for present-day and historical samples. D-statistics' were estimated for each of the NA blues, compared to an Antarctic blue, as they are from distinct genetic clusters identified by the PCA and TREEMIX analysis. Additionally, blue-fin hybrids have been reported mostly from the NA and the North Pacific (Pampoulie et al., 2020) and not from Antarctica making the Antarctic sample better for comparisons with respect to introgression analysis in blue whales. The minke whale (SRR896642, Yim et al. 2014) was the outgroup. The whole genome sequences aligned to the masked autosomal NA blue whale assembly were used in this analysis. The ABBA/BABA tests, where "A" is the ancestral allele and "B" is the derived allele, were performed in ANGSD. The sites were filtered for quality score of $>20$ and mapping quality $>30$. The SNPs from the historical samples were further filtered to remove deaminated cytosine residues using the -rmTrans parameter. The jackknife procedure was used for standard error estimations. Similarly, to study blue-humpback (Tollis et al., 2019) whale and blue-sei (SRR5665645) whale introgression, the analyses were conducted for (((Antarctic Blue, NA Blue), Humpback), Minke) and (((Antarctic Blue, NA Blue), Sei), Minke).

To detect the direction of gene flow and quantify introgression the statistic Dfoil (Pease \& Hahn, 2014) was employed. Dfoil is a five-taxon test and the phylogenetic relationship tested here was (((Sei, NA Blue), (Fin, Humpback)), Minke). The Dfoil analyses included VCF format files from whole genome sequences of blue, sei (SRR5665645), fin, humpback (Tollis et al., 2019) and minke (SRR896642) whales aligned to masked autosomal NA blue whale assembly that were processed as described above. The SNPs were filtered for missing $>0.50,>10$ bases apart, quality score of $>30$, mapping quality $>30$, coverage depth of between $3 \mathrm{X}$ and 130X and MAF $>0.1$ using VCFTOOLS and PLINK (Purcell et al., 2007). The fin whale used in the introgression analysis was also tested against another known fin whale to verify its genetic identity, which was consistent with the PCA analysis (Fig.2B) wherein it clustered with the other seven NA fin whales.

Dfoil analyses with a phylogeny of (((NA Blue 1, NA Blue 2), (Fin, Humpback)), Minke) were also examined. The historical whale samples and a low coverage sample were not included in Dfoil analysis due to shallow genome coverage.

\section{Results}

\subsection{Genome assembly.}

The NA blue whale genome of 2.49 Mbp DNA was assembled with paired-end Illumina HiSeq X ( $\sim 110 \mathrm{X})$ and Pacbio ( $\sim 50 \mathrm{X})$ sequencing technologies using DNA from a female blue whale (NW-M6, Table 1) that washed ashore in Newfounland in 2014 (Fig. 1). The assembly had a total of 11,400 contigs and a N50 of 
$1.46 \mathrm{Mb}$ (L50 of 449). The completeness of the assembly was assessed using BUSCO (Simão et al., 2015) analysis that showed $94.8 \%$ complete genes and $2.6 \%$ fragmented genes of the 4,104 reference mammalian single-copy genes tested. The 255 sex chromosome linked contig was identified as well as one contig aligned to the blue whale mitochondrial DNA (Árnason \& Gullberg, 1993). The size of the genome was estimated from Illumina reads to be ${ }^{\sim} 2.7 \mathrm{~Gb}$ indicating $92.6 \%$ of genome assembled.

Upon scanning the blue whale genome for repeats, we identified that $46.2 \%$ of the genome was composed of repetitive elements. The most abundant transposable elements were LINES (Long interspersed nuclear elements) comprising $23.21 \%$ of the genome and SINEs (Short interspersed nuclear elements) constituting about $6.86 \%$ of the genome. The other repeat elements included LTR (Long terminal repeats, $6.05 \%$ ), DNA elements $(3.53 \%)$, unclassified $(0.05 \%)$, small RNA (3.70\%), satellites (3.98\%), simple repeats (1.00\%), low complexity sequences $(0.2 \%)$ and $1.33 \%$ de novo repeats.

Transcriptome assembly annotation predicted 30,867 protein coding genes and 25,736 genes were functionally annotated (Fig. 1). The assessment of the quality of the annotation showed that $65.7 \%$ of predicted proteins had known Pfam (Finn et al., 2010) domains.

\subsection{Positive selection}

Comparison of the genomes of the blue whale, the largest whale, with one of the smallest baleen whales, the minke whale identified several oncogenes undergoing positive selection. Using a pairwise positive selection assay of single gene orthologs (SGO), we identified 72 positively selected SGO in NA blue whales. Positively selected genes included; apoptotic signalling pathway (GO:0097190); programmed cell death (GO:0097468, GO:0010421, GO:0097190) genes XBP1, C17ORF47, PTH, SCG2 and PAWR ; genes that may have a role in the evolution of large body size such as the positive regulation of cell population proliferation (GO:0008284), growth genes (GO:0040007) and cell adhesion related genes. The positive regulator of cell population proliferation genes under positive selection included PDCD10 and CGA; growth genes CCL28, TROAP and $S T R C$; and cell adhesion related genes BASP1 , GAP43 , NPPA and CRLF3 . Further examination of the positively selected genes revealed that 69 of the 72 genes identified are associated with somatic mutation in cancer cells (COSMIC v90 fasta file, Forbes et al., 2015; also see Supplementary Information).

We further evaluated genomic regions under positive selection in the 12 present-day NA blue whales using Tajima's D, and identified 52 additional genes under positive selection (Supplementary Information). The functional pathway analysis of the genes identified enrichment of nicotinic acetylcholine receptor (nAChR) signaling pathway, a pathway that regulates neurotransmitters that control cancer growth (Schuller, 2009) and genes, MYO6 , MYO1C and MYO10 (P00044) involved in hearing (Adamek, Geeves, \& Coluccio, 2011; Heimsath et al., 2017; Roux et al., 2009). The enrichment of these genes can impact acoustic communication and may signal adaptation to low frequency sound amplification and detection in blue whales.

Functional analysis of positively selected genes also identified CEACAM1 (Takeuchi et al., 2019) and HAVCR2 (Gayden et al., 2018) which are involved in negative regulation of response to tumor cells (GO:0002835). Positively selected genes in the NA blue whales included GNAS, a regulator of insulin secretion in the mTOR signaling pathway which is involved in growth and diseases of aging (Blagosklonny et al., 2013).

\subsection{Population structure}

We further wanted to investigate and visualize the genetic relationship of NA blue whales with whole genome sequences and mitochondrial genomes.

\subsubsection{Principal component analysis}

Our initial PCA performed on blue, fin and sei whales which grouped into distinct clusters when Principal Component 1 (PC1) and PC2 were plotted (Fig. 2B). One of the historical samples (NWa-CM1) was intermediate between the blue and fin whale cluster indicating it was a blue-fin hybrid which was also confirmed with D-statistics test. Two other historical samples (NWa5 and NWa6) also showed significant fin whale introgression from D-statistics analysis and were removed from further population studies. 
The second PCA using LASER was performed for blue whale samples that did not show high fin whale introgression (Fig. 2C). Most NA samples clustered together, with the Antarctica sample being distinct on PC1. PC2 indicated that two blue whale samples from NEA (NE-Ar and NE3) and NWA (NW-M6 and NW9) were outliers to the main cluster of NA samples, which can be attributed to genomic variability within the NA samples and the low total variability accounted by PC2. PC2 accounted for $\sim 9 \%$ of total variability of reference samples analyzed whereas PC1 accounted for $\sim 16 \%$ of total variability.

\subsubsection{Treemix}

The genetic relationship visualized using TREEMIX (Fig. 2D) indicated that all of the NA blue whales were allied and distinct from the Antarctic blue whale. The NEA and the NWA blue whales formed a single group. Four of the NWA blue whales (NW-M6, NWa-R4, NWa3 and NWa4) were more closely related to each other than to the other NA blue whales. The Antarctic blue whale did not share significant alleles with any of the present-day NA whales as indicated from the lack of migration events between the Antarctic and any of the NA blue whales. The migration events show gene flow between NW-M6 to NW9 and NE-Ar to NE71, which indicates that blue whales share more alleles with individuals within the same geographic regions. The gene flow indicated by the migration events between the whales were also confirmed using D-statistics.

\subsubsection{Admixture}

The NGSADMIX analysis reaffirmed that many samples (Fig. 2E) from the NA show evidence of admixture. The Antarctic sample clustered with the NWA when two ancestral populations were considered $(\mathrm{k}=2)$, which was also observed in the mitochondrial network analysis. The analysis with $\mathrm{k}=3$ populations indicated that many of the NA blue whales have mixed ancestry. The $\mathrm{k}=3$ analysis suggested that all of the blue whale historical samples had a common ancestry, which can be a possible effect of post-mortem damage not corrected despite application of methods to remove deaminated sites.

\subsubsection{Fst}

Genetic divergence estimated by the Weir and Cockerham weighted Fst suggested low (0.0093) differentiation between NEA and NWA blue whales.

\subsubsection{Mitochondrial Network Analysis}

The median joining network analysis using mitochondrial genomes and a larger sampling of historical whales produced similar results to the nuclear sequences in showing an absence of clustering of haplogroups based on the NA geographic origin of the samples (Fig. 3). Additionally, there appears to be continuity through time as haplogroups are shared between two of the four present-day NWA samples with whales killed in the same waters over a century ago. The Antarctic and South Atlantic whales cluster within the NA blue whales closest to the NWA blue whale NW-M6. Global geographic structuring using a larger sampling of whales (154) but a smaller amount of sequence (mitochondrial control region) was weak or nonexistent (Fig. S1). 
A)

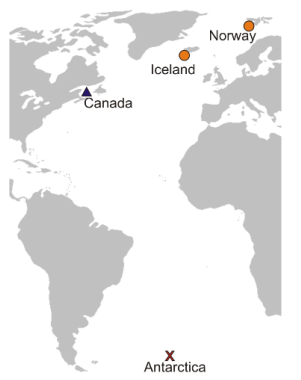

D)

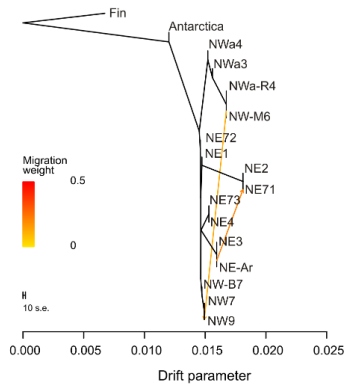

B)

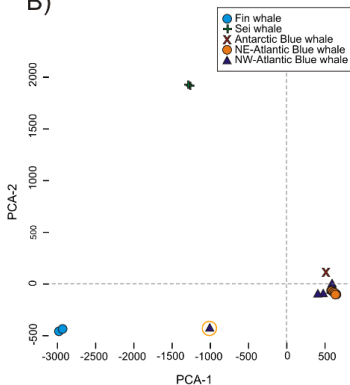

E)

Blue Whales

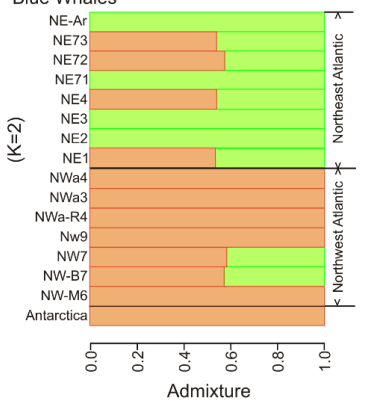

C)
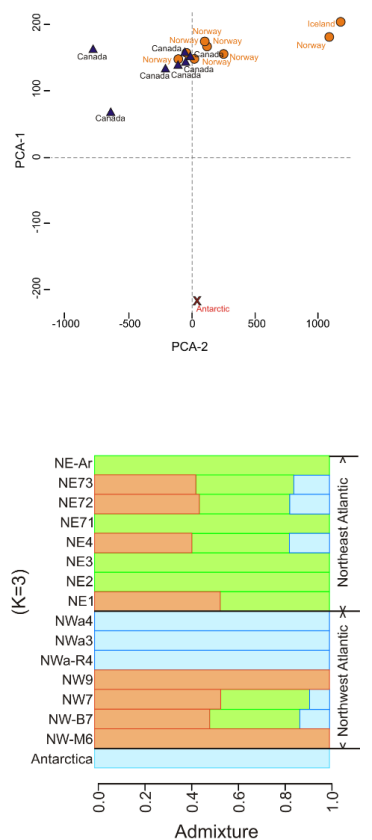

Figure 2. Population structure of present-day and historical blue whales from North Atlantic A . Map of sampling locations of blue whales B. Principal component analysis (PCA) ten Northwest Atlantic (NWA) blue whales, eight Northeast Atlantic (NEA) blue whales, one Antarctic blue whale, seven fin whales from North Atlantic and two sei whales using LASER. The circled sample represents the blue-fin hybrid historical sample (NWa-CM1) C. PCA of seven NWA blue whales, eight NEA blue whales and one Antarctic blue whale with LASER.D. Maximum likelihood tree using TREEMIX for NE, NW and Antarctic blue whales with two migration edges, considering each individual as a separate population and fin whale as the root. The horizontal branch lengths are proportional to genetic drift along that branch and the scale bar shows ten times the average standard error of the entries in the sample covariance matrix. E. Individual admixture proportions using NGSadmix for a single run from 10 runs of $\mathrm{k}=2$ and $\mathrm{k}=3$ for present-day and historical sample from NWA, NEA and Antarctic). The X-axis displays the proportion of ancestry and the Y-axis displays blue whale samples. 


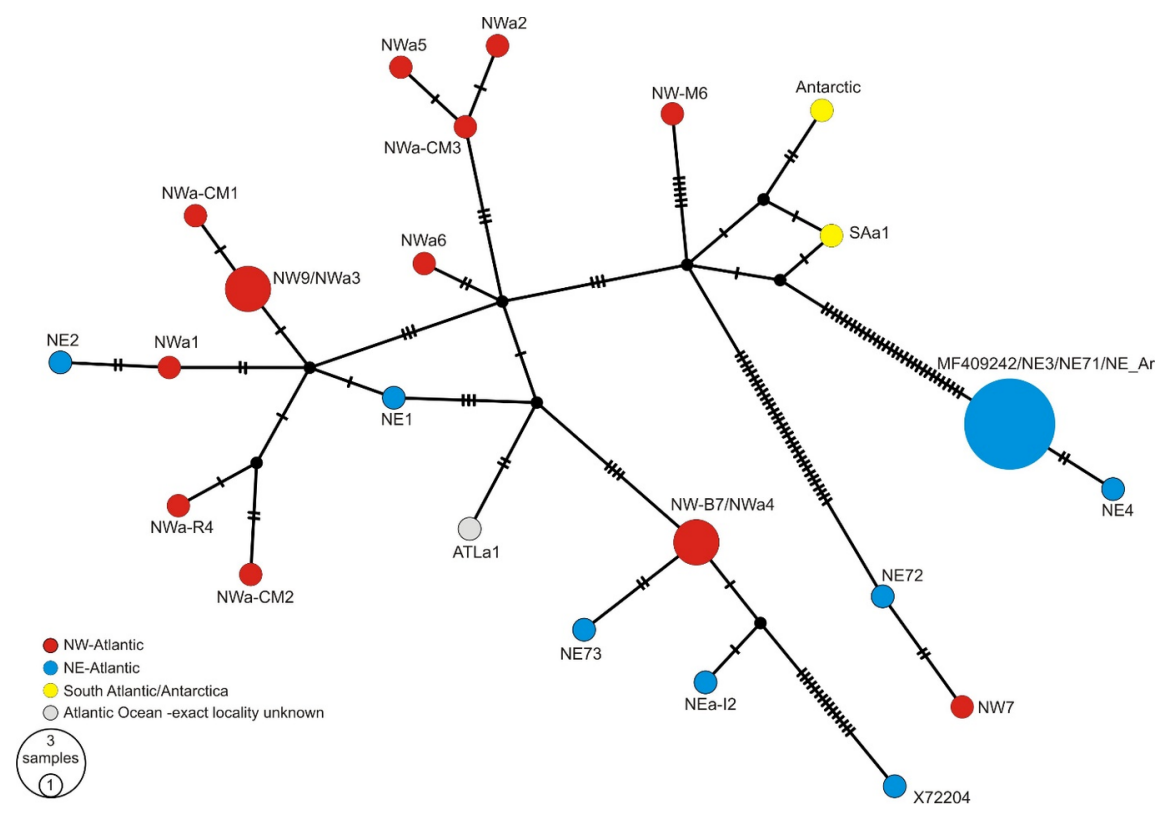

Figure 3. Median-joining network for blue whale mitochondrial genome haplotypes. Haplotypes are coloured according to the region of the Atlantic Ocean where the whale was sampled. The number of ticks denote nucleotide differences between haplogroups.

\subsubsection{Heterozygosity and population history of NA blue whales}

Genome-wide heterozygosity for the ten present-day samples $(>\sim 20 \mathrm{X})$ and the historical sample from Antarctica $(\sim 6 \mathrm{X})$ was high. Genetic variation within the NA population was also high $\sim 0.0025$ (other whale species have 0.001 (Árnason et al., 2018)). The Antarctic blue whale had the highest heterozygosity within our samples (0.0053).

Our PSMC results suggest that blue whale population size was relatively stable until approximately 400,000 years ago, when it began a decline that continued during the subsequent glacial and interglacial. There is also a suggestion of a precipitous decline during the latest glacial maximum (approximately 14,000 to 115,000 years ago). The trajectory of the fin whale population size through time is distinct from the blue whales, in that there is an initial decline beginning with the onset of the Pleistocene epoch approximately 2.5 million years ago, and a population rebound coinciding roughly with the onset of the decline in blue whales. The rebound ended and the fin whale population began to decline again during the interglacial $\sim 220,000$ years ago. The population then plateaued shortly after the onset of the last glacial maximum. 


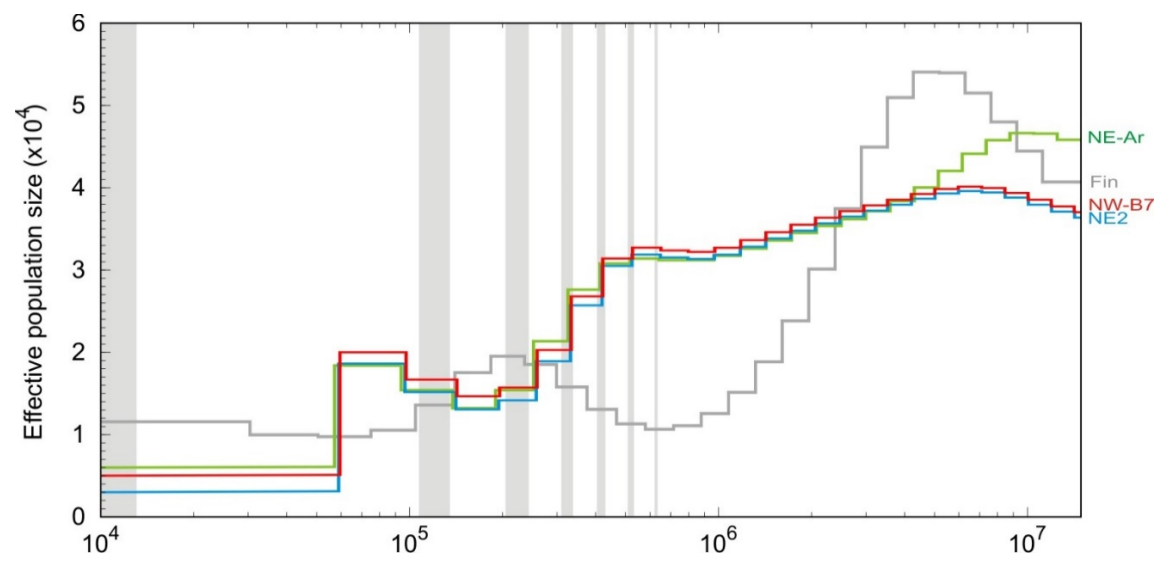

Figure 4. Inferred effective population sizes through time estimated from three blue whales (NWA and NEA whales and NE-Ar) and a fin whale using the PSMC analyses on genome sequences. The $\mathrm{X}$-axis displays time (years) going backward from the present (log scale) and the Y-axis denotes the effective population size. The plot is scaled by a generation time of 30.8 years for the blue whales and 25.9 years for the fin whale with a mutation rate of $1.39 \times 10^{-8}$ substitutions per nucleotide per generation (Árnason et al., 2018). Grey shaded areas demarcate interglacial periods in years before present: $0-14,000 ; 115,000-130,000 ; ~ 220,000-243,000$; $\sim 320,000-337,000$; and 400,000-424,000.

\subsubsection{Introgression}

All NA blue whale samples showed significant introgression (Z-score $>3$ ) with fin whales (Table 2). Four of six historical blue whale samples also showed evidence of introgression with the fin whale genome. The late twentieth century sample, NWa-CM1 (1974), had a high D-statistic value (0.94), indicating that it originated from a recent hybridization between a fin and blue whale. The mitochondria of this whale indicated that the hybrid had a blue whale mother. Two other early-1900s bone samples (NWa5 and NWa6) also had high D-statistics values, 0.54 and 0.64 respectively, which indicate hybridization in the more distant past. Blue whales from NA and the humpback whale failed to show significant interspecific introgression. Likewise, all NA blue whales, except for NE-Ar (an Icelandic sample sequenced in an earlier study (Árnason et al., 2018)), failed to show introgression with the sei whale.

Unidirectional gene flow from fin whale to blue whale was detected via Dfoil statistics in all present-day blue whale samples (Supplementary Information, Table S1). The gene flow from fin whales accounted for $\sim 3.5 \%$ of the present-day blue whale genome.

Table 2. D-statistics analysis to detect presence of gene flow between the blue and fin whales with the four-taxon phylogeny (((Antarctic Blue, NA Blue), Fin), Minke)

\begin{tabular}{lll}
\hline North-Atlantic Blue & D-Stat (SE) & Z-score \\
\hline NW-M6 & $0.035( \pm 0.004)$ & $8.04^{*}$ \\
NW-B7 & $0.033( \pm 0.004)$ & $7.67^{*}$ \\
NW7 & $0.027( \pm 0.004)$ & $6.50^{*}$ \\
NW9 & $0.031( \pm 0.004)$ & $7.08^{*}$ \\
NWa3 & $0.019( \pm 0.005)$ & $3.74^{*}$ \\
NWa4 & $0.005( \pm 0.005)$ & 0.93 \\
NWa5 & $0.57( \pm 0.006)$ & $99.56^{*}$ \\
NWa6 & $0.64( \pm 0.006)$ & $102.88^{*}$ \\
NWa-CM1 & $0.94( \pm 0.0006)$ & $1386.90^{*}$ \\
NWa-R4 & $0.014( \pm 0.005)$ & 2.80
\end{tabular}




\begin{tabular}{lll}
\hline North-Atlantic Blue & D-Stat $(\mathrm{SE})$ & Z-score \\
\hline NE1 & $0.036( \pm 0.004)$ & $8.16^{*}$ \\
NE2 & $0.049( \pm 0.004)$ & $11.25^{*}$ \\
NE3 & $0.032( \pm 0.004)$ & $7.55^{*}$ \\
NE4 & $0.048( \pm 0.004)$ & $11.01^{*}$ \\
NE71 & $0.016( \pm 0.004)$ & $3.59^{*}$ \\
NE72 & $0.035( \pm 0.004)$ & $8.09^{*}$ \\
NE73 & $0.033( \pm 0.004)$ & $7.57^{*}$ \\
NE-Ar & $0.017( \pm 0.002)$ & $7.54^{*}$ \\
\hline
\end{tabular}

\section{Discussion}

Genomic approaches are gaining importance in conservation biology. Lower costs of sequencing and improvements in data analysis in non-model organism has made these genetic tools more accessible. We present a gold-standard draft genome of a NA blue whale assembled using integrated second generation short-read and third generation long-read sequencing data. The genome was used herein to discern for the first time the population structure and demographic history of blue whales in the NA using samples from present-day whales and whales collected in the early 1900's through the $20^{\text {th }}$ century, with the earliest samples representing the blue whale population at the onset of industrial whaling. Additionally, we investigated genes that appear to be under positive selection in blue whales.

Our analysis of whole genome and mitochondrial DNA from present-day (latitudes $\sim 44^{\circ} \mathrm{N}$ to $79^{\circ} \mathrm{N}$ ) and historic samples revealed low population structuring among NA blue whales, indicating that the NA blue whales comprise a single panmictic population. This is in contrast to evidence gathered from satellite tracking and observational studies (Lesage et al., 2017; Ramp \& Sears, 2013, Silva et al., 2013), and points to the complexities of studying large marine mammals in situ and the importance of whole genome analyses to better understand population structure. The full mitochondrial analysis suggests that the historical samples from the South Atlantic and Antarctica are not as genetically distinct from North Atlantic whales as nuclear DNA analyses suggest. Further studies, with larger sample sizes, particularly for Antarctic blue whales are needed including nuclear and mitochondrial DNA analyses allowing us to test different aspects of population demography

We observed relatively high genome-wide heterozygosity in present-day NA blue whales and one historical Antarctic whale sample, consistent with reports of high heterozygosity observed in Chilean and other Antarctic whales (Torres-Florez et al., 2014). Despite being hunted to the brink of extinction, with the population size being reduced by more than $96 \%$, blue whales have retained high genetic variability. Thus, possible longterm effects of severe bottlenecking may not be fully evident in extant populations. High genetic diversity within the species is promising for species recovery, if low population numbers do not persist for an extended period of time. Similar high genetic diversity has been observed in other species that have experienced extreme overexploitation, such as Antarctic fur seals, but have since begun to recover through conservation efforts (Paijmans et al., 2020). Our examination of the demographic history of NA blue whales modeled on genome wide heterozygous sites found a decline in blue whale numbers in the North Atlantic over the last several hundreds of thousands of years during multiple periods of glaciation. The PSMC methodology loses resolution in relatively recent time periods and thus does not reveal the impact of $19^{\text {th }}$ and $20^{\text {th }}$ century whaling. Our results are similar to observations from an earlier study by Árnason et al., (2018), although use of our blue whale de novoassembly allowed for a better estimation of the effective population size at the end of the last glaciation providing an estimate of what the NA blue whale population size may have been prior to whaling.

Blue and fin whales are the two largest animals on earth and can hybridize even though they are not sibling species ( $~ 8.35$ million years since divergence) (Árnason et al., 2018). Interspecific hybridization can occur naturally or result from disruption of breeding due to anthropogenic effects. Hybridization with introgression 
can in some cases lead to the extinction of distinct species through swamping of the genome of one of the hybrid pairs. We demonstrated gene flow between blue and fin whales in all of our present-day and four (of six) historical samples; two whales from the early 20th century lacked introgression. While our sample size is very limited, this possible increase in the rate of introgression might be related to reduced mate choice in blue whales due to their reduced population size after industrial whaling. This hypothesis should be further tested with larger sample sizes of whales from pre- and post-whaling. The gene flow that we recorded between fin whales and NA blue whales was not evident in previous studies (Árnason et al., 2018; Westbury et al., 2019) that were done without the blue whale genome as a reference. The direction of gene flow was fin to blue whale in all present-day blue whales analyzed from NA, consistent with the observation of several interspecific hybrids resulting from mating of a male fin whales and female blue whales (Pampoulie et al., 2020).

We also assembled and annotated the NA blue whale transcriptome, allowing for functional analysis of positively selected blue whale genes. We identified several $\mathrm{nAChR}$ signaling pathway genes that are associated with cancer development (Schuller, 2009) and also the selection of genes involved in apoptosis. Earlier studies of suppression of cancer in marine mammals detected positive selection of another apoptosis pathway gene (ETNK1 ) in humpback whales (Tollis et al., 2019) and positive selection of genes regulating nucleotide repair, chromatin structure and transcription in bowhead whales (Keane et al., 2015). Sperm whale gene analysis has revealed several cancer genes (MMP7, CASP12 and PRSS33 ) that were positively selected (Warren et al., 2017). Diseases like cancer which are associated with age and large body size, surprisingly do not affect large aquatic mammals (Keane et al., 2015; Tollis et al., 2019). The gene pathways involved are not fully understood. Our analysis indicates functional enrichment of genes in the nAChR pathway that might further elucidate the underlying mechanism of cancer suppression. This might be a useful tool in medical research.

In summary, the results from our study suggest that the blue whales in the NA comprise a single population. Thus, future conservation assessment and planning should be conducted in a holistic framework viewing NA blue whales as a single population. Hybridization of blue whales with fin whales is frequent in the NA and introgression might be increasing in the present population. Future rates of introgression should be monitored. The genetic variability in NA blue whales is high and the slow recovery of blue whales in the NA is not associated with reduction of heterozygosity via bottlenecking in extant populations. However, it may become a factor in the future if populations do not increase from their current low levels. Rates of introgression with fin whales should be monitored. Further studies with a larger sample size for present-day and historical blue whales from around the world could be used to examine changes in levels of heterozygosity and resolve questions regarding gene flow among populations of blue whales from different oceans to enhance conservation planning efforts.

\section{Acknowledgments}

Thanks to Lois A. Harwood and Jack Lawson (Department of Fisheries and Oceans) for alerting us to the presence of the blue whale strandings in Newfoundland and for facilitating the process of allowing us to recover the whales. Many thanks to the people of Trout River, Rocky Harbour and Woody Point who were instrumental in partnering with us to salvage the two blue whales that are central to this study. The authors also acknowledge the support of Tonya Wimmer (Marine Animal Rescue Society) for blue whale tissues and Kamal Khidas (Canadian Museum of Nature), Michael McGowen, Darrin Lunde and John Ososky (Smithsonian National Museum of Natural History) for allowing us to sample their museum collections, and Veronica Chevasco in assisting in optimizing protocols for ancient DNA recovery. Additionally, we thank Hanne Garmel (The Whaling Museum, Sandefjord, Norway), Haakon Livland (Slottsfjellsmuseet, Tønsberg, Norway), and Hanneke Meijer (University Museum Bergen, Norway) for help in providing historical samples and David William Peter Dolan (Dept. of Informatics, University of Bergen) for bioinformatic/infrastructure support. The genome sequencing and bioinformatic analysis was made possible by the generous support of the Alan and Patricia Koval Foundation, The Centre for Applied Genomics at The Hospital for Sick Children, CanSeq150 by Canada's Genomics Enterprise (a Canada Foundation of Innovation Major Science Initiative 
award), and a computing grant 'Resource for Research Groups' from Compute Canada (2018-2020). The Norwegian Research Council ICE-whales programme (no. 244488/E10) and Norwegian Polar Institute supported collections of NE Atlantic blue whale tissues. The sequencing activities at the Genomics Core Facility of the University of Bergen was supported by the Fram Center Hazardous Substances Flagship Program (grant no. 602018), the Research Council of Norway (grant no. 245979/F50) and the Trond Mohn Foundation (grant no. BFS2016-genom). The bioinformatic analysis at the University of Bergen was provided through ELIXIR Norway, Research Council of Norway grant 270068 (to the University of Bergen, the University of Oslo, the Arctic University of Norway in Troms $\varnothing$, the Norwegian University of Science and Technology and the Norwegian University of Life Sciences: NMBU). The funding for salary of S.J. was provided by a Rebanks post-doctoral fellowship and the Koval foundation. S.W.S. was funded by the GlaxoSmithKline Endowed Chair in Genome Sciences at the Hospital for Sick Children and University of Toronto. L.L. is currently funded by the Lap-Chee Tsui Fellowship for Research Excellence, though this work was started while she was at the ROM.

\section{References}

Adamek, N., Geeves, M. A., \& Coluccio, L. M. (2011). Myo1c mutations associated with hearing loss cause defects in the interaction with nucleotide and actin. Cellular Molecular Life Sciences,68, 139-150. Altschul, S. F., Madden, T. L., Schäffer, A. A., Zhang, J., Zhang, Z., Miller, W., Lipman, D. J. (1997). Gapped BLAST and PSI-BLAST: a new generation of protein database search programs. Nucleic Acids Research, 25, 33893402. Árnason, Ú., \& Gullberg, A. (1993). Comparison between the complete mtDNA sequences of the blue and the fin whale, two species that can hybridize in nature. Jourmal of Molecular Evolution, 37, 312-322. Árnason, Ú., Lammers, F., Kumar, V., Nilsson, M. A., \& Janke, A. (2018). Whole-genome sequencing of the blue whale and other rorquals finds signatures for introgressive gene flow. Science Advances, 4 , eaap9873. Bandelt, H. J., Forster, P. \& Röhl, A. (1999). Median-joining networks for inferring intraspecific phylogenies. Molecular Biology Evolution, 16, 37-48. Blagosklonny, M. V. (2013). Big mice die young but large animals live longer. Aging, 5, 227-233. COSEWIC. 2012. COSEWIC status appraisal summary on the Blue Whale Balaenoptera musculus, Atlantic population, in Canada. Committee on the Status of Endangered Wildlife in Canada. Ottawa.xii pp. www.registrelep-sararegistry.gc.ca/default Díez-del-Molino, D., Sánchez-Barreiro, F., Barnes, I., Gilbert, M. T. P., \& Dalén, L. (2018). Quantifying temporal genomic erosion in endangered species. Trend in Ecology \&3 Evolution, 33, 176-185. Edgar, R. C. (2004). MUSCLE: multiple sequence alignment with high accuracy and high throughput. Nucleic Acids Research, 32, 1792-1797. Ewart, K. M., Johnson, R. N., Ogden, R., Joseph, L., Frankham, G. J., \& Lo, N. (2019). Museum specimens provide reliable SNP data for population genomic analysis of a widely distributed but threatened cockatoo species. Molecular Ecology Resources, 19, 1578-1592. Finn, R. D., Tate, J., Mistry, J., Coggill, P. C., Sammut, S. J, Hotz, H. R., ... Bateman, A. (2010). The Pfam protein families database.Nucleic Acids Research, 38, D211-D222. Foote, A. D., Martin, M. D., Louis, M., Pacheco, G., Robertson, K. M., Sinding, M.-H. S., Amaral, A. R., Baird, R.W., Baker, C.S.... Morin, P. A. (2019). Killer whale genomes reveal a complex history of recurrent admixture and vicariance. Molecular Ecology, 28, 3427-3444. Forbes, S. A., Beare, D., Gunasekaran, P., Leung, K., Bindal, N., Boutselakis, H., ... Campbell, P. J. (2015). COSMIC: exploring the world's knowledge of somatic mutations in human cancer. Nucleic Acids Research, 43, D805-D811. Gayden, T., Sepulveda, F. E., Quang, D. A. K., Pratt, J., Valera, E. T., Garrigue, A., ... Jabado, N. (2018). Germline HAVCR2 mutations altering TIM-3 characterize subcutaneous panniculitis-like $\mathrm{T}$ cell lymphomas with hemophagocytic lymphohistiocytic syndrome. Nature Genetics,50, 1650-1657. Grabherr, M. G., Haas, B. J., Yassour, M., Levin, J. Z., Thompson, D. A., Amit, I., ... Regev, A., (2011). Trinity: reconstructing a full-length transcriptome without a genome from RNA-Seq data.Nature Biotechnology, 29, 644-652. Green, R. E., Krause, J., Briggs, A. W., Maricic, T., Stenzel, U., Kircher, M. .. Paabo, S. (2010). A draft sequence of the Neanderthal genome. Science, 328, 710-722. Haubold, B., Pfaffelhuber, P., \& Lynch, M. (2010). MIRho - A program for estimating the population mutation and recombination rates from shotgun-sequenced diploid genomes. Molecular Ecology, 15,356. Heimsath, E. G. J., Yim, Y., Mustapha, M., Hammer, J. A., \& Cheney, R. E. (2017). Myosin-X knockout is semi-lethal and demonstrates that myosin-X functions in neural tube closure, pigmentation, hyaloid vasculature regression, and filopodia formation. Scientific Reports, 7, 17354. 
Hohenlohe, P. A., Funk, W. C, \& Rajora, O. P. (2020). Population genomics for wildlife conservation and management. Molecular Ecology, 30, 62-82. Holt, C., \& Yandell, M. (2011). MAKER2: an annotation pipeline and genome-database management tool for second-generation genome projects.BMC Bioinformatics, 12, 491. Keane, M., Semeiks, J., Webb, A. E., Li, Y. I., Quesada, V., Craig, T., .. Magalhaes, J. P. (2015). Insights into the evolution of longevity from the bowhead whale genome. Cell Reports, 10, 112-122. Koren, S., Schatz, M. C., Walenz, B. P., Martin, J., Howard, J. T., Ganapathy, G., .. Phillippy, A. M. (2012). Hybrid error correction and de novo assembly of single-molecule sequencing reads. Nature Biotechnology, 30, 693-700. Korneliussen, T. S., Albrechtsen, A., \& Nielsen, R. (2014). ANGSD: Analysis of next generation sequencing data. BMC Bioinformatics, 15, 356. Langmead, B., \& Salzberg, S. L. (2012). Fast gapped-read alignment with Bowtie 2. Nature Methods, 9, 357. Leduc, R. G., Archer, F. I., Lang, A. R., Martien, K. K., Hancock-Hanser, B. Torres-Florez, J. P., .. Taylor, B. L. (2017). Genetic variation in blue whales in the eastern pacific: Implication for taxonomy and use of common wintering grounds. Molecular Ecology,26, 740751. Leigh, J. W., \& Bryant, D. (2015). POPART: full-feature software for haplotype network construction. Methods in Ecology and Evolution,6, 1110-1116. Lesage, V., Gavrilchuk, K., Andrews, R. D. \& Sears, R. (2017). Foraging areas, migratory movements and winter destinations of blue whales from the western North Atlantic. Endangered Species Research,34, 27-43. Li, H., \& Durbin, R. (2009). Fast and accurate short read alignment with Burrows-Wheeler transform. Bioinformatics, 25, 1754-1760. Li, H. \& Durbin, R. (2012). Inference of human population history from whole genome sequence of a single individual. Nature, 475, 493496. McDonald, M. A., Mesnick, S. L., \& Hildebrand, J. A. (2006). Biogeographic characterization of blue whale song worldwide: Using song to identify populations. Journal of Cetacean Research and Management, 8, 55-65. Meyer, W. K., Jamison, J., Richter, R., Woods, S. E., Partha, R., Kowalczyk, A., .. Clark, N. L. (2018). Ancient convergent losses of Paraoxonase 1 yield potential risks for modern marine mammals.Science 361, 591-594. Mikheyev, A., Tin, M. M. Y., Arora, J., \& Seeley, T. D. (2015). Museum samples reveal rapid evolution by wild honeybees exposed to a novel parasite. Nature Communications, 6, 7991. Miller, M. R., Brunelli, J. P., Wheeler, P. A., Liu S., Rexroad 3 ${ }^{\text {rd }, ~ C . ~ E ., ~ P a l t i, ~ Y ., ~ . . . ~ T h o r g a a r d, ~ G . ~ H . ~(2012) . ~ A ~}$ conserved haplotype controls parallel adaptation in geographically distant salmonid populations. Molecular Ecology, 21, 237-249 Nieukirk, S. L., Stafford, K. M., Mellinger, D. K., Dziak, R. P. \& Fox, C. G. (2004). Low-frequency whale and seismic airgun sounds recorded in the mid-Atlantic Ocean. Journal of Acoustic Society of America,115, 1832-1843. Okada, H., \& Mak, T. W. (2004). Pathways of apoptotic and nonapoptotic death in tumour cells. Nature Reviews Cancer, 4, 592-603. Paijmans, A. J., Stoffel, M. A., Bester, M. N., Cleary, A. C., De Bruyn, P. J. N., Forcada, J., .. Hoffman, J. I. (2020). The genetic legacy of extreme exploitation in a polar vertebrate. Scientific Reports, 10, 5089. Pampoulie, C., Gislason, D., Olafsdottir, G., Chosson, V., Halldorsson, S. D., Marianiet, S., .. Vikingsson, G. A. (2020). Evidence of unidirectional hybridization and second-generation adult hybrid between the two largest animals on Earth, the fin and blue whales.Evolutionary Applications, 00, 1-8. Pease, J. B., \& Hahn, M. W. (2014). Detection and polarization of introgression in a five-taxon phylogeny. Systematic Biology,64, 651-662 Pickrell, J., \& Pritchard, J. (2012). Inference of population splits and mixtures from genome-wide allele frequency data. PLoS Genetics, 8, e1002967. Pike, D. G., Gunnlaugsson, T., Mikkelsen, B., Halldorsson, S. D. \& Vikingsson, G. A. (2019). Estimates of the abundance of cetaceans in the Central North Atlantic based on the NASS Icelandic and Faroese Shipboard surveys conducted in 2015. NAMMCO Scientific Publications 11. Purcell, S., Neale, B., Todd-Brown, K., Thomas, L., Ferreira, M. A. R., Bender, D., .. Sham, P. C., (2007). PLINK: a toolset for whole-genome association and population-based linkage analysis. American Journal of Human Genetics, 81, 559-575. Ramp, C. \& Sears, R. Distribution, densities, and annual occurrence of individual blue whales (Balaenoptera musculus) in the Gulf of St. Lawrence, Canada from 1980-2008. DFO Can. Sci. Advis. Sec. Res. Doc. 2012/157. vii + 37 p (2013). Rocha, R. C., Clapham, P. J., \& Ivashchenko, Y. V. (2014). Emptying the oceans: a summary of industrial whaling catches in the 20th century.Marine Fisheries Review, 76, 37-48. Rosenbaum, H. C., Egan, M., Clapham, P. J., Brownell, R. L. Jr, \& DeSalle, R. (1997) An effective method for isolating DNA from historical specimens of baleen. Molecular Ecology, 6, 677-681. Roux, I., Hosie, S., Johnson, S. L., Bahloul, A., Cayet, N., Nouaille, S., .. Safieddine, S. (2009). Myosin VI is required for the proper maturation and function of inner hair cell ribbon synapses. Human Molecular Genetics, 18, 4615-4628. Sabeti, P. C., Schaffner, S. F., Fry, B., Lohmueller, J., Varilly, P., Shamovsky, O., 
... Lander, E. S. (2006). Positive natural selection in the human lineage. Science, 312, 1614-1620. Sears, R., \& Perrin, W. F., (2018). "Blue Whale: "Balaenoptera musculus" in Encyclopedia of marine mammals, B. Wursig, and J. G. M. Thewissen, Eds. (Academic Press), pp. 110-114. Sinding, M.-H. S., Gopalakrishan, S., Vieira, F. G., Samaniego, J. A. C., Raundrup, K., Jorgensen, M. P. H., .. Gilbert, M. T. P. (2018). Population genomics of grey wolves and wolf-like canids in North America. PLoS Genetics, 14, e1007745. Silva, M. A., Prieto, R., Jonsen, I., Baumgartner, M. F., \& Santos, R. S. (2013). North Atlantic blue and fin whales suspend their spring migration to forage in middle latitudes: building up energy reserves for the journey? PloS One, 8, e76507. Simao, F. A., Waterhouse, R. M., Ioannidis, P., Kriventseva, E. V., \& Zdobnov, E. M. (2015). BUSCO: assessing genome assembly and annotation completeness with singlecopy orthologs. Bioinformatics,31, 3210-3212. Skotte, L., Korneliussen, T. S., \& Albrechtsen, A. (2013). Estimating individual admixture proportions from next generation sequencing data.Genetics, 195, 693-702. Schuller, H. M. (2009). Is cancer triggered by altered signalling of nicotinic acetylcholine receptors? Nature Reviews Cancer,9, 195-205. Storrie, L., Lydersen, C., Andersen, M., Wynn, R.B., \& Kovacs, K. M. (2018). Determining the species assemblage and habitat use of cetaceans in the Svalbard Archipelago, based on recorded observations from 2002-2014. Polar Research, 37, 1463065. Takeuchi, A., Yokoyama, S., Nakamori, M., Nakamura, M., Ojima, T., Yamaguchi, S., ... Yamaue, H. (2019). Loss of CEACAM1 is associated with poor prognosis and peritoneal dissemination of patients with gastric cancer. Science Report, 9, 12702. Tebbutt, S. J., Stewart, R. E., \& Hill, D. F. (2000). Isolation and characterisation of DNA from whale bone. Journal of the Royal Society of New Zealand, 30, 365-371. Thomas, P. O., Reeves, R. R., \& Brownell, R. L. (2016). Status of the world's baleen whales. Marine Mammal Science, 32, 682-734. Tollis, M., Robbins, J., Webb, A. E., Kuderna, L. F. K., Caulin, A. F. ... Maley, C. C. (2019) Return to the sea, get huge, beat cancer: an analysis of cetacean genomes including an assembly for the humpback whale (Megaptera novaeangliae). Molecular Biology and Evolution, 36, 1746-1763. Torres-Florez, J. P., Hucke-Gaete, R., Rosenbaum, H., Figueroa, C. C. (2014). High genetic diversity in a small population: the case of Chilean blue whales. Ecology Evolution, 4, 1398-1412. Trapnell, C., Pachter, L. \& Salzberg, S. L. (2009). TopHat: discovering splice junctions with RNA-Seq. Bioinformatics, 25, 1105-1111. Wang, C., Zhan, X.,Liang, L., Abecasis, G. R., \& Lin, X. (2015). Improved ancestry estimation for both genotyping and sequencing data using projection Procrustes analysis and genotype imputation.American Journal Human Genetics, 96, 926-937. Wang, D., Zhang, Y., Zhang, Z., Zhu, J., \& Yu, J. (2010). KaKs_Calculator 2.0: a toolkit incorporating gamma-series methods and sliding window strategies. Genomics Proteomics Bioinformatics,8, 77-80. Warren, W. C., Kuderna, L., Alexander, A., Catchen, J., Perez-Silva, J. G., Lopez-Otin, C., ... Wise Sr, J. P. (2017). The novel evolution of the sperm whale genome. Genome Biology and Evolution, 9, 3260-3264. Webb, A. E.,Walsh, T. A., \& O'Connell, M. J. (2017). VESPA: very large-scale evolutionary and selective pressure analyses. PeerJ Computer Science, 3, e118. Weir, B. S. \& Cockerham, C. C. (1984). Estimating F-Statistics for the analysis of population structure. Evolution, 38, 1358-1370. Westbury, M. V., Petersen, B., \& Lorenzen, E. D. (2019) Genomic analyses reveal an absence of contemporary introgressive admixture between fin whales and blue whales, despite known hybrids. PloS One, 14, e0222004. Yim, H. S., Cho, Y., Guang, X., Kang, S. G., Jeong, J. Y., Cha, S. S. .. Lee, J. H. (2014). Minke whale genome and aquatic adaptation in cetaceans. Nature Genetics, 46, 88-92.

\section{Author contributions:}

The study was conceived and supervised by M.D.E. Sample collection by M.D.E., B.K.L., J.M., A.G., R.L., K.M.K., C.L. \& H.R. Genome and transcriptome assembly, annotation, introgression, heterozygosity and population analysis was done by S.J., PSMC and mitochondrial analysis by O.H. \& Tajima's D analysis by L.L. Guidance on genome assembly and analysis was provided by S.W.S. \& S.L. Manuscript written by S.J. \& O.H. All the authors commented upon the final manuscript.

\section{Data Accessibility:}

All sequence data are in the process of being submitted to NCBI under the title "Balaenoptera musculus (Blue Whale) and Balaenoptera physalus (Fin Whale) Genome sequencing and assembly". We are awaiting accession numbers for the whales in this study and will provide them once received. 